\title{
Percepciones sobre la calidad y las tarifas del servicio de agua potable de los usuarios de Ciudad de México
}

\author{
Quality and fares of water supply service \\ perceptions for users in Mexico City
}

\section{Percepções em torno da qualidade e as tarifas do serviço de água encanada nos usuários da Cidade do México}

\author{
José Marcos Bustos Aguayo* \\ Margarita Juárez Nájera** \\ Francisco Rubén Sandoval Vázquez ${ }^{* * *}$ \\ María Luisa Quintero Soto**** \\ Cruz García Lirios ${ }^{* * * * *}$ \\ Fecha de recepción: 6 de marzo de 2017 \\ Fecha de evaluación: 17 de agosto de 2017 \\ Fecha de aceptación: 15 de setiembre de 2017 \\ Fecha de publicacion en linea: 1 de diciembre de 2017 \\ DOI: http://dx.doi.org/10.18359/reds.3236
}

Cómo citar este artículo:

Bustos Aguayo, J.M., Juárez Nájera, M., Sandoval Vázquez, F. R., Quintero Soto, M.L. y García Lirios, C. (2017). Percepciones sobre la calidad y las tarifas del servicio de agua potable de los usuarios de Ciudad de México. Revista Educación y Desarrollo Social, 11(2), 20-31. DOI: org/10/18359/reds.3236.


trónico: quinluisa@yahoo.com.mx

Universidad Nacional Autónoma de México (UNAM), Facultad de Estudios Superiores de Zaragoza, Ciudad de México. Correo electrónico: marcos.bustos@unam.mx

** Universidad Autónoma Metropolitana, Iztapalapa, Ciudad de México.

Correo electrónico:mjn@correo.azc.uam.mx

*** Universidad Autónoma de Morelos, Facultad de Psicología, Cuernavaca, Morelos, México. Correo

***** Universidad Autónoma del Estado de México, Huehuetoca, Estado de México. Correo electrónico: cgarcial213@profesor.uaemex.mx 


\section{Resumen}

La calidad y las tarifas de servicios municipales son un tema central en la agenda de desarrollo local y gobernanza de la formación para la sustentabilidad, pero la literatura correspondiente parece destacar la relación entre la identidad y el entorno como determinantes de una evaluación del sistema de gestión hídrica estatal y la administración del servicio público correspondiente. De esta manera, el objetivo del presente artículo fue establecer un modelo para el estudio de la percepción de la calidad y las tarifas de servicios municipales, a fin de poder destacar la importancia de las diferencias y similitudes entre los gobernantes y los gobernados en el marco de la escasez de agua, la contaminación de los mantos acuíferos y la sobreexplotación de los cuerpos de agua. Se realizó un estudio documental con una selección no probabilística de fuentes indexadas a repositorios nacionales, considerando el periodo de publicación de 2000 a 2017, así como la inclusión de las palabras clave, siguiendo la técnica Delphi para la especificación del modelo. Se advierte una línea de investigación comparativa entre los factores esgrimidos en la revisión de la literatura.

Palabras clave: recursos hídricos, servicios públicos, gobernanza, tarifas, abastecimiento, modelo.

\section{Abstract}

The quality and fares of the county utilities are the main topic in the local development and public agenda of sustainability development, but the relevant literature seems to highlight the relationship between identity and the environment as determinants of the evaluation of the water management system and the administration of the public utility. In this sense, the objective of the present article was to establish a model to study of the perception of the quality and the fares on county services; in order to be able to highlight the importance of differences and similarities between the authorities and the people in the context of the lacking of water, the contamination of water wells and the over-exploitation of water resources. A documentary study was carried out with a non-probabilistic selection of sources indexed to national archives, considering the publication period from 2000 to 2017, as well as including keywords, following the Delphi method for model specification. A line of comparative research between the factors used in the review of the literature is shown.

Keywords: Water resources, public utilities, government authorities, fares, utility supply, model.

\section{Resumo}

A qualidade e as tarifas dos serviços municipais são um tema central na agenda de desenvolvimento local e governança do treinamento para a sustentabilidade, mas a literatura correspondente parece destacar a relação entre a identidade e o meio ambiente como determinantes de uma avaliação do sistema de gestão hídrica do Estado e a administração do serviço público correspondente. Desta forma, o objetivo do presente artigo foi estabelecer um modelo para o estudo da percepção da qualidade e das tarifas dos serviços municipais, com o fim de poder enfatizar a importância das diferenças e semelhanças entre os governantes e os governados no quadro da escassez de água, a poluição dos aquíferos e a sobre-exploração dos corpos de água. Um estudo documental foi realizado com uma seleção não probabilística de fontes indexadas a repositórios nacionais, considerando o período de publicação de 2000 a 2017, bem como a inclusão das palavras-chave, seguindo a técnica Delphi para a especificação do modelo. É notada uma linha de pesquisa comparativa entre os fatores usados na revisão da literatura.

Palavras-chave: recursos hídricos, serviços públicos, governança, tarifas, abastecimento, modelo. 


\section{Introducción}

La percepción de la calidad del servicio, para los fines del presente escrito, alude a un sistema de procesamiento de información delimitado por los datos disponibles de escasez o abundancia del recurso, o bien, la reducción o aumento del costo del servicio, ambos indicadores en función de los valores, creencias, disposiciones, intenciones y estilos de consumo de los usuarios de este (Aguilar et al., 2015).

La percepción de la calidad del servicio ha sido un fenómeno abordado teniendo en cuenta la relación que guarda el costo y los beneficios esperados, así como los riesgos por la demora del pago o las amenazas de una baja calidad y sus efectos en los estilos de vida y consumo. Es así como la percepción de la calidad del servicio al ser contrastada con índices de calidad devela asimetrías significativas a partir de las cuales pueden establecerse criterios de evaluación de las políticas de servicios y la satisfacción de los usuarios (Carreón et al., 2013).

Empero, los marcos teóricos, conceptuales y empíricos solo han considerado los servicios como públicos, susceptibles de ser privados y con un antecedente comunitario, soslayando las propuestas de gobernanza o administración consensuada entre usuarios y autoridades en cuanto a tarifas que correspondan a la calidad del 22 servicio municipal (Carreón et al., 2015).

Incluso, en un sentido de establecimiento de consensos y corresponsabilidades, la literatura consultada advierte una aproximación multicultural dominante que consiste en la tolerancia como estrategia de gestión. Es decir, los grupos que dominan, como los gobernantes, implementan un sistema de tandeo acorde a las demandas de los usuarios, pero inhibiendo sus expectativas o propuestas para mejorar la calidad del servicio (Carreón, Hernández y García, 2016).

Sin embargo la percepción de los usuarios, al estar sesgada en cuanto a necesidades y expectativas, centrada en la corrupción y sin considerar el costo real que supone la gestión y la administración de los servicios públicos, cancela toda posibilidad de diálogo entre las partes en conflicto y los actores políticos y sociales (Carreón et al., 2014).

Es menester explorar un cogobierno de los servicios públicos en el que la gestión estatal no impere como una rectoría y la participación social no solo se concentre en las demandas y expectativas sin asumir el costo real del servicio, excluyendo los subsidios gubernamentales y las condonaciones acordadas entre las partes (García, 2005).

Precisamente, el objetivo del presente trabajo es establecer un modelo para el estudio de la percepción de la calidad de los servicios públicos, con énfasis en el sistema de abastecimiento y cobro del agua potable. Se hizo un trabajo no experimental, documental, exploratorio y transversal con una selección muestral no probabilística de fuentes indexadas a dos repositorios líderes de México 
como Latindex y Redalyc, así como al periodo de publicación y la inclusión de palabras clave como criterios de la búsqueda informativa. Los datos fueron procesados siguiendo la técnica Delphi, la cual advierte que una integración de los contenidos está en función de su comparación, estableciendo los ejes y trayectorias de las variables en un modelo.

El proyecto se inscribe en la disciplina de Trabajo Social, área de Estudios Documentales, e incluye conceptos provenientes de la psicología social, la sociología urbana y la economía social. El proyecto fue financiado por la Universidad Nacional Autónoma de México, Dirección General de Asuntos del Personal Académico, Programa de Apoyo a Proyectos de Investigación e Innovación Tecnológica, número de registro IN305516.

\section{Teoría de las percepciones de la calidad del servicio}

Los marcos teóricos y conceptuales que explican la percepción de la calidad del servicio son: 1) teoría de la calidad de vida, 2) teoría del bienestar subjetivo, 3) teoría de la satisfacción de vida, y 4) teoría de la fiabilidad social.

La percepción de la calidad del servicio, desde la óptica de la teoría de la calidad de vida, refiere a una dimensión subjetiva centrada en satisfactores como el acceso, la utilidad y el costo. El usuario que disfruta de ellos se formará una expectativa positiva de sus autoridades y evaluará de manera favorable a la administración correspondiente (García, 2006).

Empero, tal escenario no explica el grado de satisfacción que el usuario tiene con respecto al servicio público, razón por la cual sus percepciones de bienestar destacan como indicadores de una baja, nula o alta calidad (García, 2011).

La teoría del bienestar subjetivo, a diferencia de la teoría de la calidad del servicio, sostiene que el grado de satisfacción será determinante en una imagen y evaluación positiva de quienes administran el servicio público, pero tal efecto depende de las emociones del usuario (García et al., 2015e). Si el usuario desarrolla expectativas de bienestar no significa que esté satisfecho con el servicio público. Esto es así porque las emociones oscilan entre la insatisfacción y la satisfacción, y al no haber criterios universales de medición, varían en cada caso particular o grupal (García, 2012).

Por consiguiente, la teoría de la satisfacción de vida vendrá a proponer que una evaluación positiva del servicio público depende más de la experiencia significativa del usuario para con el servicio público que de una imagen o representación. De este modo, a diferencia de la aproximación del bienestar subjetivo que cuestiona en torno al grado de satisfacción, desde el enfoque de la satisfacción de vida se pregunta sobre la experiencia y se infiere si esta ha sido significativa o no (García, 2007). 
De este modo, un usuario satisfecho es aquel que declara haber tenido una experiencia significativa con el servicio público. Es el caso de quienes están conscientes de haber hecho un gasto de recursos y reconocen que el costo por tal estilo de vida es justo o corresponde a sus estimaciones (García, 2008).

La satisfacción de vida, asumida como una experiencia significativa, denota una empatía y confianza del usuario para con las autoridades gestoras y administradoras del servicio público. Se trata de una fiabilidad social que se observa en el pago en tiempo y forma del servicio público, o bien, en la solicitud de ampliación o extensión del servicio, así como en la reducción significativa de quejas, sugerencias o reclamos (García et al., 2017).

La teoría de la fiabilidad social destaca la satisfacción del usuario, su experiencia significativa, así como su percepción sesgada de mejoría del servicio, o bien, la medición objetiva de la calidad de este y por ende de la calidad de vida y el bienestar subjetivo, pero soslaya su capacidad de no solo emitir juicios, evaluaciones o recomendaciones, sino además de ser partícipe en la transparencia y la rendición de cuentas de la administración pública y del desempeño de sus funcionarios (García, Carreón y Hernández, 2016).

La gobernanza de los servicios públicos se distingue por su grado de comunicación bidireccional, motivación horizontal, decisión consensuada y participación corresponsable. En ese sentido, la especificación de un modelo permitirá explicar y anticipar un nuevo sistema de cogobierno, cogestión, coadministración y corresponsabilidad entre los actores políticos y sociales (García et al., 2012).

\section{Estudios de las percepciones de la calidad del servicio}

Se revisan los estudios de la calidad y el coste del servicio de agua con base en la psicología de la sustentabilidad, reportados desde 2000 a la fecha, con la intención de explorar dos dimensiones preponderantes: las expectativas de abastecimiento hídrico gratuito y de la calidad del servicio de abastecimiento. Sin embargo líneas de investigación alusivas a la comparación entre ambas, tanto en escenarios electorales como libres de proselitismo y contienda política, suponen la emergencia de percepciones de utilidad (García et al., 2013).

La medición de las expectativas de los usuarios con respecto al recorte del suministro y el incremento de tarifas llevadas a cabo por sus autoridades locales, supone que los usuarios son agentes principales en los conflictos con las autoridades. Es menester explicar las trayectorias de las relaciones de dependencia entre las variables esgrimidas en la literatura con la finalidad de anticipar escenarios de boicots, tomas de unidades de abastecimiento, confrontaciones con la policía, cierre de avenidas y movilizaciones colectivas (García et al., 2014c). 
Los estudios psicológicos de la sustentabilidad hídrica han centrado su interés en la predicción de un comportamiento favorable al uso sustentable del agua, es el caso de los motivos de ahorro como determinantes del cuidado de esta, o bien, las emociones positivas como antecedentes de su bajo consumo. La psicología de la sustentabilidad hídrica ha también observado correlaciones entre las expectativas de riesgo y utilidad del servicio de abastecimiento respecto al pago oportuno del recibo. Incluso, la aceptación y pago de un sistema de cobro y tarifas acorde a la calidad del servicio es también explicado por las expectativas del usuario (Vilchis, García, Carreón, Hernández et al., 2017).

Sin embargo los estudios psicológicos de la sustentabilidad hídrica también han demostrado un alto grado de desconfianza de los usuarios respecto a las políticas de abastecimiento y cobro de sus gobiernos locales. En ese sentido, las expectativas sobre el costo y la calidad del servicio serían determinantes no solo para el ahorro de agua, sino también para el pago del recibo (García et al., 2015b). De este modo, la psicología de la sustentabilidad hídrica ha demostrado que las expectativas de riesgo están asociadas al cuidado del agua y las de utilidad están más próximas a su derroche (García et al., 2014b).

En el marco de la gestión y administración de los recursos y servicios hídricos, los estudios psicológicos de la sustentabilidad han demostrado que cuando las autoridades ejercen estrategias de abastecimiento intermitente combinadas con un incremento en el consumo de agua, propician su ahorro en zonas de ingreso económico medio, generan la solidaridad y cooperación en las de bajo ingreso económico y una demanda creciente en aquellas con altos ingresos (Hernández et al., 2014).

En el caso de las zonas con ingreso económico medio, los usos y costumbres locales son factores que medían el impacto del recorte en el suministro de agua a las comunidades y barrios periféricos. De esta manera, las expectativas de la calidad del servicio de agua potable y el sistema de cobro subsecuente son influidas por los valores y las normas de consumo locales (García, 2015a). Esto es así porque se supone que la disponibilidad de recursos hídricos corresponde al servicio de abastecimiento público. Es decir, si los cuerpos de agua están disponibles a los consumidores, entonces estos tienden a considerar que el suministro es un derecho que el Estado debe garantizar, extrayendo, potabilizando y distribuyendo continua y gratuitamente el agua a la ciudadanía (Limón et al., 2017).

A pesar de que la psicología de la sustentabilidad ha establecido que las expectativas de los usuarios respecto a la calidad del servicio y el sistema de cobro son determinantes en el ahorro y pago del servicio, los conflictos entre usuarios y autoridades se han exacerbado en la medida en que la calidad del agua es baja y su costo es alto (Pérez et al., 2017). 
Los estudios psicológicos de la sustentabilidad hídrica también han demostrado que los conflictos entre autoridades y usuarios son antecedente de la violencia y delincuencia local, ya que mientras la calidad del servicio disminuye y se incrementan sus costos, la ciudadanía desconfía aún más de ellas, y además se organiza para la autogestión, excluyéndolas de sus decisiones y acciones de autoabastecimiento en el mejor de los casos, mientras otro sector menos organizado genera acciones de protesta, boicots a redes de suministro, secuestro de unidades de abastecimiento, bloqueos de avenidas, denuncia de fugas y confrontaciones con las fuerzas del orden (Sánchez et al., 2017).

La prensa de circulación nacional, en su seguimiento a los conflictos por la gestión y administración de los recursos y servicios hídricos, crea desesperanza entre sus lectores en la medida en que atribuye la responsabilidad a las autoridades y a los usuarios. Las audiencias que siguen las noticias locales en torno a las fugas de agua son menos dadas a denunciar las irregularidades de las redes de suministro locales y residenciales que aquellas que siguen las noticias globales de desastres naturales, inundaciones y sequías (García, Carreón y Hernández, 2016).

En ambos estudios, las expectativas de la calidad del servicio de abastecimiento y las tarifas de las unidades de consumo se tratan como indicadores que reflejan la relación entre autoridades y usuarios respecto al servicio público de agua (García et al., 2014a).

Las expectativas de los usuarios para con las decisiones y acciones gubernamentales en cuanto al recorte del suministro y el incremento de tarifas develan relaciones de poder en las que ambos actores no solo están inmersos, sino además condicionan sus posibilidades de gestión y administración de los recursos y servicios hídricos (Carreón et al., 2015c).

Las diferencias entre autoridades y usuarios se observan en los conflictos y la violencia, pero ambas se cristalizan en el derroche de agua y la morosidad en el pago del servicio. Tal proceso se intensifica en la medida en que se aproximan elecciones políticas (García et al., 2015d). Es decir, las expectativas de los usuarios en relación con el abastecimiento y cobro del servicio son instrumento de gestión y administración de los recursos y servicios hídricos en periodos electorales, durante los comicios y después de la contienda (Limón et al., 2017).

Las relaciones teóricas, conceptuales y empíricas revisadas en el estado del conocimiento se vincularán con las observaciones y mediciones de un estudio empírico, con una muestra de una localidad durante un periodo electoral (García, 2008).

Empero, si las relaciones teóricas, conceptuales y empíricas relativas a las expectativas de los usuarios son diferentes a los resultados obtenidos, entonces se 
considerará que la calidad y el sistema de tarifas del servicio hídrico son fenómenos diferentes que pertenecen a dimensiones de gestión y administración sin vínculo alguno con las expectativas de los usuarios, y por ende lejanas a la anticipación de escenarios de conflicto (García, 2005).

Las expectativas de los usuarios respecto al abastecimiento intermitente y el incremento de las tarifas suponen la continuidad del sistema de abastecimiento y cobro del servicio y parecen evidenciar que se circunscriben a la calidad y al costo. Los usuarios no solo diferencian ambas cuestiones, la calidad y las tarifas, sino que además las vinculan a escenarios posibles de consumo en función de las estrategias de abastecimiento y cobro de las autoridades (Carreón et al., 2015).

Las expectativas de abastecimiento y de cobro parecen ser un diagnóstico sesgado de las estrategias de abastecimiento y sus efectos en la economía y los estilos de vida residenciales. A este tenor, la literatura consultada advierte que al ser considerada como un bien público, el agua está condenada a ser auspiciada por el Estado mediante un sistema de aumento de tarifas, subsidios y condonaciones (García et al., 2014b).

Si se considerara como un bien común, el agua determinaría un cuidado permanente sin importar la calidad y el coste, ya que la sola idea de bien común influiría en su respeto y cuidado tal y como ocurre con los patrimonios de la sociedad civil (Pérez et al., 2017).
Las expectativas son un constructo que explica y anticipa escenarios de conflictos entre autoridades locales y usuarios domésticos ante la calidad y el costo del servicio de agua potable. Las expectativas de abastecimiento y de cobro son dos dimensiones desde las que se observan tendencias preponderantes de desencuentros entre los actores políticos y sociales, sus expectativas e intenciones que conformarán escenarios de guerra por la gestión y administración de los recursos hídricos en sus distintas modalidades y disponibilidades (García et al., 2013).

\section{Modelo de las percepciones de la calidad del servicio}

La representación de los ejes y las trayectorias de relaciones entre los factores esgrimidos en los marcos teóricos, conceptuales y empíricos es conocida como establecimiento de un modelo (véase figura 1).

La percepción del servicio hídrico, indicado por las tarifas, el suministro, las condonaciones y los subsidios esperados, supone un proceso de cogestión y coadministración, enfocado en la proximidad de las instituciones estatales para con las organizaciones civiles. En ese sentido, se trata de un sistema de cogobierno de los recursos hídricos fundamentado en considerar el agua como un bien común, el cual debe conservarse para construir un futuro compartido (hipótesis 1).

Por consiguiente, las tarifas percibidas reflejarían un consenso en torno al cual usuarios y autoridades se comprometen 


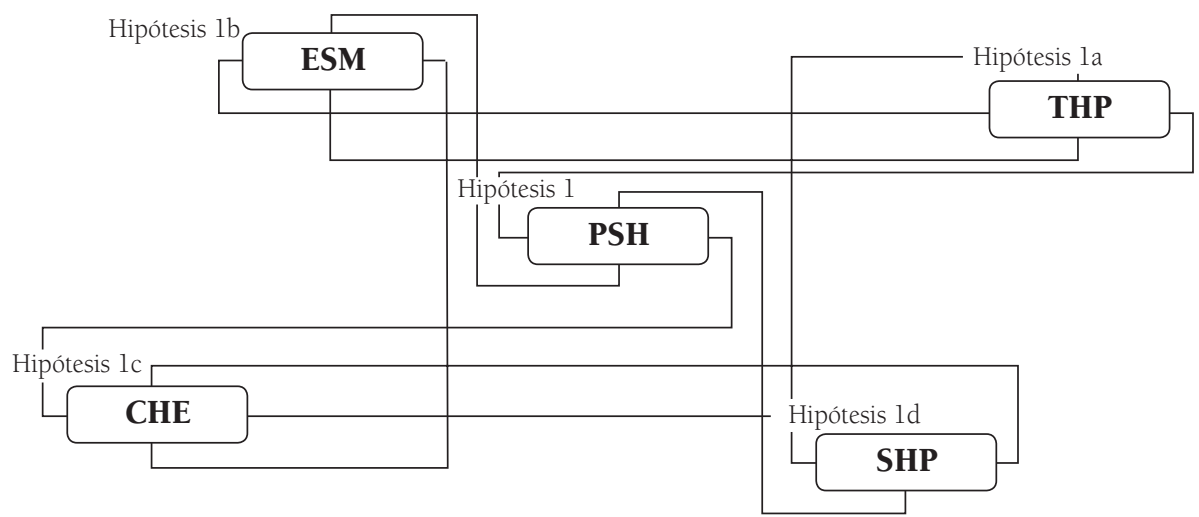

Figura 1. Modelo perceptual de la calidad del servicio hídrico público

PSH: percepción del servicio hídrico; THP: tarifas hídricas percibidas; ESM: expectativas de suministro municipal; CHE: condonaciones hídricas esperadas; SHP: subsidios hídricos percibidos.

Fuente: elaboración propia.

a establecer un precio justo y acorde a estándares de calidad. En contraste, la ausencia de tales índices de calidad supone la hegemonía del Estado o de la sociedad civil organizada como gestora del abastecimiento y el cobro del servicio de agua potable, alcantarillado y tratamiento (hipótesis la).

Precisamente, en torno al abastecimiento hídrico, la calidad percibida estaría reflejada en la aceptación o el rechazo del tandeo, un sistema de distribución austero y solidario entre quienes reciben un mayor volumen y quienes carecen de acceso a tal ventaja comparativa (hipótesis 1b).

Empero, el sistema de tarifas al estar vinculado a una política de subsidio a colonias populares que destinan hasta el $20 \%$ de sus ingresos a la compra de garrafones de agua potable, supone un debate público y abierto entre los actores sociales y estatales con la finalidad de establecer acuerdos de reducción de estos subsidios y ajustes al costo de la calidad del servicio, más que a las necesidades o las expectativas locales (hipótesis 1c).

Por último, en cuanto a la condonación como estrategia de seguridad local ante posibles brotes de violencia por el abastecimiento de agua, traducidos en secuestros de pipas, boicots a tomas principales, confrontaciones verbales o físicas, plantones o manifestaciones de acción colectiva, es menester una negociación intensiva a fin de poder regularizar el suministro y alcanzar un sistema de cobro acorde a los casos más apremiantes (hipótesis 1d). 


\section{Consideraciones finales}

El aporte del presente trabajo al estado del conocimiento radica en el establecimiento de un modelo para el estudio de la percepción de la calidad del servicio de agua potable, considerando cuatro indicadores que explicarían las diferencias y similitudes entre los actores políticos y sociales involucrados en la gestión, pero con un sentido de corresponsabilidad que permita alcanzar la gobernanza del sistema.

Sin embargo el tipo de selección muestral informativa y los criterios de elección limitan el aporte de un contexto local, el cual puede ser extendido si se considera la inclusión de fuentes internacionales como Ebsco, Scopus o Copernicus y una técnica de procesamiento de información más sofisticada como la minería de textos.

En cuanto a los marcos teóricos, conceptuales y empíricos que destacan la percepción como factor sustancial de las diferencias entre la gestión estatal y la autogestión social, el presente trabajo ha propuesto que se integren ambas estrategias a fin de poder establecer un cogobierno legitimado por factores que develen las asimetrías entre usuarios y autoridades.

La investigación en torno a la comparación de ambas percepciones, estatales y sociales, permitirá anticipar escenarios de conflictos y cambios sociales. Ello supondría la emergencia de un sistema de gobernanza o administración consensual que legitimaría al sistema en su totalidad y se podría alcanzar un sistema de cobro acorde a la calidad del servicio como a las necesidades y las expectativas sociales.

Por último, en cuanto a los trabajos de revisión de García et al., (2017), Limón et al., (2017), Pérez et al., 2017) y Sánchez et al., (2017) en los que se destaca la identidad en relación con el entorno como determinantes de una formación civil protectora del medio ambiente, el presente trabajo plantea la importancia de los sistemas de abastecimiento y tarifario, los cuales reflejarían las diferencias y similitudes entre los usuarios del servicio y las autoridades.

Es menester un estudio comparativo entre escenarios, identidades y sistemas de abastecimiento y cobro para establecer los determinantes específicos del desarrollo y la gobernanza de los servicios municipales, principalmente antes, durante y después de celebrados los comicios locales para establecer las diferencias y similitudes entre los actores y en función de tales condicionantes.

\section{Referencias}

Aguilar, J. A., Carreón, J., García, C., Hernández, J. y Rosas, F. J. (2015). Gobernanza de actitudes sociopolíticas. Perspectivas Rurales, 14 (27), 107-148.

Carreón, J., Hernández, J., Morales, M. L., Rivera, B. L., Limón, G. A. y García, C. (2013). Actitudes y construcción de la agenda pública. Realidades, 3(2), 91-105. 
Carreón, J., Hernández, J., García, C., Bustos, J., Morales, M. y Aguilar, J. (2014). La psicología de la sustentabilidad hídrica. Políticas públicas y modelos de consumo. Aposta, 63, 1-29.

Carreón, J., Hernández, J., Morales, M. L. y García, C. (2014). Complejidad sociopolítica del transporte público. Tlatemoani, 15, 119-140.

Carreón, J., Hernández, J., Morales, M. L. y García, C. (2014). Intenciones de voto a favor de propuestas verdes. ¿Nuevo paradigma ambiental y posmaterialismo social? Revista de Psicología Política, 12 (32), 37-56.

Carreón, J., Bustos, J. M., García, C., Hernández, J. y Mendoza, D. (2015). Utilización de SPSS y AMOS en un estudio del pensamiento ambientalista y las intenciones de voto en una muestra de estudiantes. Multidisciplina, 20, 7695.

Carreón, J., Hernández, J. y García, C. (2016). Agenda pública y participación sociopolítica. Fermentum, 75(26), 41-56.

García, C. (2005). Los estudios psicológicos de la sustentabilidad. Mneme, 6(13), 1-63.

García, C. (2006). El problema de la sustentabilidad. Enfoques, 18(2), 145158.

García, C. (2007). Una aproximación sistémica de la realidad política. Sincronía, 12(44), 1-10.

García, C. (2008). Un estudio sobre la representatividad sostenible. Entelequia,

García, C. (2011). Las estructuras de la sustentabilidad. Tecsistecalt, 3(10), 1-19.

García, C. (2012). Las disertaciones estructurales ambientales. Entelequia, 14, 171-184.

García, C., Montero, M. E., Bustos, J. M., Carreón, J. y Hernández, J. (2012). Sistemas de democracia sustentable. Comunitaria, 4, 123-156.

García, C., Morales, M. L., Bustos, J. M., Carreón, J., Limón, G. A. y Hernández, J. (2013). Fundamentos sistémicos de la complejidad política. Enfoques, 25 (1), 7-23.

García, C., Carreón, J., Hernández, J. Montero, M. y Bustos, J. M. (2014a). Sistemas sociopsicológicos de la propaganda sustentable. Enseñanza e Investigación en Psicología, 19(1), 219244.

García, C., Carreón, J., Mecalco, J., Hernández, J., Bautista, M. y Méndez, A. (2014b). Sistemas políticos complejos: Implicaciones para la seguridad pública sustentable. Acciones e Investigaciones Sociales, 34, 183-216.

García, C., Carreón, J., Rosas, F. J., Aguilar, J. A. y Hernández, J. (2014c). Teorías para explicar el origen, construcción y establecimiento de la agenda sociopolítica. Perspectivas, 25, 241-263.

García, C., Carreón J., Bustos, J. M., Hernández, J. y Salinas, R. (2015a). Especificación de un modelo de comunicación de riesgos ambientales ante el cambio climático. Entre Ciencias, 3(6), 71-90.

García, C., Carreón, J., Hernández, J., Bustos, J. M., Aguilar, J. A., Rosas, F. J. y Vilchis, F. J. (2015b). Campo de poder en torno al sexismo ambivalente. Implicaciones 
$\overline{\text { Percepciones sobre la calidad y las tarifas del servicio de agua potable de los usuarios de ciudad de México }}$

para el desarrollo humano sostenible. Revista Uruguaya de Enfermería, 10 (1), 83-97.

García, C., Carreón, J., Mendoza, D., Aguilar, J. A. y Hernández, J. (2015c). Contraste de un modelo de legitimidad sociopolítica. Tendencias \& Retos, 20 (2), 121-133.

García, C., Carreón, J., Quintero, M. L. y Bustos, J. M. (2015d). Confiabilidad y validez de un instrumento que mide la gobernanza del bienestar subjetivo. Ciencia Administrativa, 2, 143-154

García, C., Carreón. J., Hernández, J. y Rivera, B. L. (2015e). Confiabilidad y validez de un instrumento que mide la dimensión sociopolítica de la calidad de vida. Psicumex, 5(2), 79-92.

García, C., Carreón J. y Hernández, J. (2016). Especificación de un modelo de complejidad sociopolítica. Revista de Psicología Política, 14(16), 40-59.

García, C., Carreón, J. y Hernández, J. (2016). Gobernanza de la seguridad pública a partir de la identidad y percepción de riesgo establecidos en la agenda ciudadana por los medios de comunicación. Rumbos, 9(13), 103-116.

García, C., Bustos, J. M., Carreón, J. y Hernández, J. (2017). Marcos teóricos y conceptuales en torno al desarrollo local. Margen, 85, 1-11.

Hernández, J., Carreón, J., Morales, M. L., Aguilar, J. A. y García, C. (2014).
Complejidad sociopolítica del transporte público. Implicaciones para el desarrollo local sustentable. Razón \& Palabra, 86, 449-468.

Limón, G. A., Rivera, B. L., Bustos, J. M., Juárez, M. y García, C. (2017). Gobernanza del cambio climático: Especificación de un modelo de actitudes hacia el cambio climático. Revista de Ciencias Política, 30, 1-31.

Pérez, M. I., Aguilar, J. A., Morales, M. L., Pérez, G. y García, C. (2017). Gobernanza de las preferencias electorales: contrastación de un modelo de intención de voto en estudiantes de una universidad púbica. Revista Internacional de Investigación en Ciencias Sociales, 13(1), 49-59.

Sánchez, A., Quintero, M. L., Sánchez, A., Fierro, E. y García, C. (2017). Gobernanza del emprendimiento social: especificación de un modelo para el estudio de la innovación local. Nómadas, 51, 1-21.

Vilchis, F. J., García, C., Carreón, J., Hernández, J., Bustos, J. M., Valdés, O. (2017). La identidad criminal en la Ciudad de México. En J. A. Gastélum (coord.), Siglo XXI: La urgencia de la sustentabilidad y la responsabilidad social (pp. 177-200). México, D. F: Universidad de occidente [UDEO]. 